\title{
A Constant Bound for the Periods of Parallel Chip-firing Games with Many Chips
}

\author{
Paul Myer Kominers and Scott Duke Kominers
}

\begin{abstract}
We prove that any parallel chip-firing game on a graph $G$ with at least $4|E(G)|-|V(G)|$ chips stabilizes, i.e. such a game has eventual period of length 1. Furthermore, we obtain a polynomial bound on the number of rounds before stabilization. This result is a counterpoint to previous results which showed that the eventual periods of parallel chip-firing games with few chips need not even be polynomially bounded.
\end{abstract}

Mathematics Subject Classification (2000). 05C35, 05C85, 68Q25 (Primary); 37B15, 68R10, 68Q80 (Secondary).

Keywords. chip-firing, parallel chip-firing, stabilization, polynomial time.

\section{Introduction}

We let $G$ be a finite, undirected, connected graph and denote the vertex and edge sets of $G$ by $V(G)$ and $E(G)$, respectively. For a vertex $v \in V(G)$, we denote the degree of $v$ by $\operatorname{deg}(v)$.

In a chip-firing game on $G$, some number of chips are distributed among the $|V(G)|$ vertices of $G$. Then, in each of a sequence of rounds $t=1,2, \ldots$, a vertex $v \in V$ with more than $\operatorname{deg}(v)$ chips is selected and fired-one chip from $v$ is moved to each of $v$ 's neighbors. The parallel chip-firing game on $G$ is defined similarly: chips are distributed among the $|V(G)|$ vertices of $G$, and in each of a sequence of rounds $t=1,2, \ldots$, all vertices $v \in V$ with more than $\operatorname{deg}(v)$ chips are fired.

The chip-firing game was first introduced by Spencer [7] for infinite graphs; Björner, Lovász, and Shor [3] extended the game's definition to general finite graphs. Terminating chip-firing games on undirected graphs have been studied

The second author gratefully acknowledges the support of a Harvard Mathematics Department Highbridge Fellowship. 
extensively and are surprisingly well-behaved. For example, the length of a terminating chip-firing game on a graph $G$ is bounded by a polynomial in the characteristics of $G$ (see [8]). Chip-firing games also have important applications; notably, they are related to Tutte polynomials (see [6]) and the critical groups of graphs (see [1]).

Bitar and Goles [2] introduced the parallel chip-firing game, observing that such a game must necessarily converge towards a periodic sequence of chip configurations. They furthermore proved that any parallel chip-firing game on a finite, undirected, connected, acyclic graph has eventual period of length at most 2 . Disproving two previous conjectures to the contrary, Kiwi et al. [4] later showed that the periods of parallel chip-firing games need not be polynomially bounded. Specifically, Kiwi et al. [4] constructed a parallel chip-firing game on an $n$-node, connected, undirected graph having eventual period of length $\exp (\Omega(\sqrt{n \log n}))$.

In this note, we show the following counterpoint to the result of Kiwi et al. [4].

Theorem 1. If $G$ is a finite, undirected, connected graph, then any parallel chipfiring game on $G$ with at least $4|E(G)|-|V(G)|$ chips has eventual period of length 1.

We show additionally that when the conditions of Theorem 1 hold, the game on $G$ converges to its period-1 chip configuration in a number of rounds bounded above by a polynomial in the characteristics of $G$.

Our approach draws from the literature on chip-firing, using in particular a key result from Tardos's [8] proof that terminating chip-firing games conclude in polynomial time.

\section{The Setting}

We denote by $\varphi_{t}(v)$ the total number times a vertex $v \in V(G)$ has fired by the end of round $t$. For consistency, we throughout denote by $c$ the total number of chips in a parallel chip-firing game on $G$.

We say that a vertex has stabilized in some round if, after that round, the number of chips on that vertex will not change throughout the remainder of the game. Thus, a parallel chip-firing game on $G$ has eventual period 1 if and only if all vertices of $G$ stabilize. Abusing terminology slightly, we therefore say that a parallel chip-firing game on $G$ stabilizes if its eventual period is 1 .

We say that a vertex $v \in V(G)$ is abundant if it holds at least $2 \operatorname{deg}(v)$ chips. Any vertex $v \in V(G)$ with $k \geq \operatorname{deg}(v)$ chips at the beginning of a round passes $\operatorname{deg}(v)$ chips to its neighbors and can, at most, receive one chip from each of its $\operatorname{deg}(v)$ neighbors. Thus, such a vertex cannot end the round with more than $k$ chips. In particular, the set of abundant vertices of $G$ can only shrink over the course of a parallel chip-firing game on $G$. 


\section{Main Theorem}

We now prove the following stabilization theorem.

Theorem 2. Let $G$ be a finite, undirected, connected graph with diameter $d$. In any parallel chip-firing game on $G$ with

$$
c \geq 4|E(G)|-|V(G)|
$$

chips, every vertex $v \in V(G)$ will stabilize within $|V(G)| \cdot d \cdot c$ rounds.

This result implies Theorem 1 of the introduction. Additionally, Theorem 2 encapsulates the $c \geq 3 n$ cases of the result of the first author [5] for parallel chipfiring games on $n$-cycles. Our methods are inspired by those of Tardos [8]; they are essentially independent of the arguments used in [5].

We use the following lemma, which is a special case of Lemma 5 of Tardos [8].

Lemma 3. Let $v, v^{\prime} \in V(G)$ be adjacent vertices of $G$. Then, $\left|\varphi_{t}(v)-\varphi_{t}\left(v^{\prime}\right)\right| \leq c$ for all $t$.

Additionally, we need an observation about the condition $c \geq 4|E(G)|-|V(G)|$.

Lemma 4. For $G$ a graph and $c \geq 4|E(G)|-|V(G)|$, in any parallel chip-firing game on $G$ with $c$ chips there is at least one vertex $v_{*} \in V(G)$ which fires every round.

Proof. It suffices to find a vertex $v_{*} \in V(G)$ which fires every round $t$ during which some vertex $v \in V(G)$ holds fewer than $2 \operatorname{deg}(v)-1$ chips.

As observed above, it is not possible for a vertex $v \in V(G)$ which is not abundant at the beginning of round $t$ to become abundant after round $t$. However, the condition

$$
c \geq 4|E(G)|-|V(G)|
$$

guarantees that whenever some $v \in V(G)$ holds fewer than $2 \operatorname{deg}(v)-1$ chips there is also at least one abundant vertex $v^{\prime} \in V(G)$. The existence of some vertex $v_{*} \in V(G)$ which is abundant in every round when some vertex $v \in V(G)$ has fewer than $2 \operatorname{deg}(v)-1$ chips then follows immediately, and we have the lemma.

We may now proceed with the proof of our main result.

Proof of Theorem 2. By Lemma 4, there is some vertex $v_{*} \in V(G)$ which fires every round. Denoting the rounds by $t=1,2, \ldots$, we then have $\varphi_{t}\left(v_{*}\right)=t$ for all rounds $t$. By Lemma 3, we then know that

$$
\left|\varphi_{t}\left(v_{*}\right)-\varphi_{t}(v)\right| \leq d \cdot c
$$

for all $t$ and $v \in V(G)$. Since $\varphi_{t}\left(v_{*}\right)$ is strictly increasing in $t$, no $v \in V(G)$ may fail to fire for more than $d \cdot c$ rounds. In the worst case, all but one of the vertices fire in each round when some vertex does not fire; hence after $|V(G)| \cdot d \cdot c$ rounds all the vertices of $G$ fire every round, and the game has stabilized. 


\section{Remarks}

\subsection{On the Exponential Period Lengths Observed by Kiwi et al. [4]}

The results of Kiwi et al. [4] show that parallel chip-firing games with sufficiently few chips can have eventual periods of exponential lengths. By contrast, our Theorem 1 shows that the period-lengths of parallel chip-firing games with sufficiently many chips are bounded by the constant 1 .

The main class of examples provided by Kiwi et al. [4] uses graphs $G_{m}$ with exactly $3\left(\sum_{i=1}^{m} p_{i}\right)+1$ vertices, $3\left(\sum_{i=1}^{m} p_{i}\right)+m$ edges, and $\underline{c}_{m}:=3\left(\sum_{i=1}^{m} p_{i}\right)+m$ chips. (Here, $m>1$ is fixed and $\left\{p_{i}\right\}_{i=1}^{\infty}$ is the ordered set of primes.) There is a substantial gap between $\underline{\mathrm{c}}_{m}$ and

$$
\bar{c}_{m}:=4\left(3 \sum_{i=1}^{m} p_{i}+m\right)-\left(3 \sum_{i=1}^{m} p_{i}+1\right)=9 \sum_{i=1}^{m} p_{i}+4 m-1,
$$

the number of chips required for a parallel chip-firing game on $G_{m}$ to be guaranteed to stabilize. The behavior of parallel chip-firing games on $G_{m}$ with $\bar{c}_{m}>c>\underline{c}_{m}$ chips has not been studied; it would be interesting to know when a polynomially bounded period length can be guaranteed. In particular, it seems surprising that the period-length bound proceeds from exponential to constant pursuant to a polynomial increase in the number of chips.

\subsection{On Possible Improvements of our Bounds}

Our Lemma 4 is, in some sense, dual to Lemma 4 of Tardos [8] which shows that for any terminating chip-firing game on $G$ there is a distinguished vertex $v_{*} \in$ $V(G)$ which never fires. Such a result need not hold when $c<4|E(G)|-|V(G)|$. Indeed, the two-vertex graph $G$ with vertex set $V(G)=\left\{v_{1}, v_{2}\right\}$ and edge set $E(G)=\left\{\left\{v_{1}, v_{2}\right\}\right\}$ provides a simple counterexample. We have

$$
4|E(G)|-|V(G)|-1=4 \cdot 1-2-1=1,
$$

while no vertex fires in every round of a single-chip parallel chip-firing game on $G$.

Nonetheless, the first author [5] has shown that the conclusion of Theorem 1 holds when $G$ is an $n$-cycle and $c \geq 3 n-2=4|E(G)|-|V(G)|-2$. Thus, the lower bound on $c$ used in Theorems 1 and 2 may be relaxed in special cases; it may be possible to relax this bound more generally.

Additionally, the "worst case" scenario examined in the proof of Theorem 2 seems unlikely to occur often when the number of chips on $G$ is large. Thus, it is likely that the $c$-dependence of the stabilization-time bound in Theorem 2 can be relaxed.

\section{References}

[1] N. L. BigGs, Chip-firing and the critical group of a graph, Journal of Algebraic Combinatorics, 9, 25-45 (1999). 
[2] J. Bitar And E. Goles, Parallel chip firing games on graphs, Theoretical Computer Science, 92, 291-300 (1992).

[3] A. BJörner, L. Lovász, And P. Shor, Chip-firing games on graphs, European Journal of Combinatorics, 12, 283-291 (1991).

[4] M. A. Kiwi ET AL., No polynomial bound for the period of the parallel chip firing game on graphs, Theoretical Computer Science, 136, 527-532 (1994).

[5] P. M. Kominers, The candy-passing game for $c \geq 3 n-2$, Pi Mu Epsilon Journal, 12, 459-460 (2008).

[6] C. M. López, Chip firing and the Tutte polynomial, Annals of Combinatorics, 1, 253-259 (1997).

[7] J. Spencer, Balancing vectors in the max norm, Combinatorica, 6, 55-65 (1986).

[8] G. TArdos, Polynomial bound for a chip firing game on graphs, SIAM Journal on Discrete Mathematics, 1, 397-398 (1988).

Paul Myer Kominers

Department of Economics, Massachusetts Institute of Technology

c/o 8520 Burning Tree Road

Bethesda, MD 20817

e-mail: pkoms@mit.edu

Scott Duke Kominers

Department of Economics, Harvard University, and Harvard Business School

Wyss Hall, Harvard Business School

Soldiers Field, Boston, MA 02163

e-mail: kominers@fas . harvard.edu 